\title{
LAS ENERGÍAS ALTERNATIVAS ¿UNA OPORTUNIDAD PARA COLOMBIA?
}

\author{
María Giraldo \\ Escuela de Negocios, Gestión y Sostenibilidad \\ Programa de Especialización en Gestión Empresarial \\ Especialista en Gestión Empresarial \\ magigi11@hotmail.com \\ Raúl Vacca Ramírez \\ Escuela de Negocios, Gestión y Sostenibilidad \\ Programa de Especialización en Gestión Empresarial \\ Especialista en Gestión Empresarial \\ vacca@enel.com \\ Andrés Urrego Quintanilla \\ Escuela de Negocios, Gestión y Sostenibilidad \\ Programa de Especialización en Gestión Empresarial \\ Especialista en Gestión Empresarial \\ andres.urregoq@gmail.com
}

\section{Resumen:}

Este artículo aporta elementos de análisis sobre el desarrollo de la energía renovable en Colombia a partir de bibliografía científica, con el objetivo de analizar las oportunidades de negocio para invertir en energías renovables alternativas. El documento está divido en nueve secciones: las tres primeras secciones presentan una explicación de los tipos de fuentes de generación de energía, las energías convencionales y alternativas, y su importancia. La cuarta sección hace un análisis de costos y beneficios tecnológicos. De la quinta a la octava sección se focaliza en Colombia, revisando la composición de la generación, la aplicabilidad de las energías alternativas, la conveniencia de la transición de energía convencional a fuentes no convencionales y las oportunidades de negocio con el uso de energías renovables no convencionales. La novena sección presenta las conclusiones.

Palabras Claves: Energía, energía renovable, energías alternativas. 


\section{Introducción:}

En Colombia el 70\% de la generación eléctrica depende de la generación hidráulica, gracias a la gran riqueza hídrica del País, Sin embargo, hoy en día la realización de grandes proyectos hidroeléctricos se ha convertido en una tarea casi que imposible por el impacto ambiental y la gran cantidad de obstáculos que deben superar para su construcción. La tendencia mundial de generación de energía eléctrica está enfocándose en las tecnologías que garantizan la sostenibilidad de nuestro planeta y por efecto directo los costos de las nuevas tecnologías han empezado a competir con las soluciones tradicionales. El objetivo de este artículo es analizar la oportunidad de negocio que tiene Colombia para invertir en energías renovables alternativas mediante el conocimiento de las fuentes de generación de energía en Colombia, la identificación de las fuentes de generación a partir de energías alternativas y de las oportunidades de negocio en Colombia derivadas de la generación de energía con fuentes no convencionales.

\section{Metodología:}

En primer lugar, se ha realizado una búsqueda bibliográfica sistemática en las bases de datos Google academic, Scielo, Redalyc y Dialnet, limitándose a los idiomas inglés y castellano y usando como descriptores los siguientes términos: negocios, oportunidades económicas y energías alternativas en Colombia. Los registros obtenidos oscilaron entre 1.300 y 56 registros tras la combinación de las diferentes palabras claves.

Finalmente hemos incluido veintinueve artículos publicados en los últimos diez años, desde el año 2007 hasta 2017, excepto un artículo publicado en 2003 que brinda una perspectiva histórica de las energías renovables en América Latina, el resto son revisiones bibliográficas acerca de la evolución, costos, beneficios y perspectivas futuras de las energías renovables que vimos conveniente incluir.

\section{Desarrollo:}

Tipos de fuentes generación de energía: El progreso de la civilización actual depende en gran medida del aprovechamiento de las fuentes de energía no renovables, las cuales históricamente se originan en los combustibles fósiles: carbón, petróleo y gas natural, de ahí las consecuencias ambientales negativas por el exceso de consumo de los combustibles y la reciente preocupación por la optimización de la eficiencia en la extracción, la transportación, el consumo, y la conservación de los recursos energéticos que han motivado la búsqueda de recursos y fuentes energéticos renovables.

En el artículo Energías Renovables y Conservación de Energía (Badii, Guillen \& Abreu, 2016; pag 142) ratifican la total dependencia del sol para la supervivencia del planeta tierra. 
El 99\% de la energía que caliente el planeta y nuestros edificios proviene del sol y no cuesta. Sin la energía solar o capital solar, la temperatura promedio del planeta sería cerca de $240^{\circ} \mathrm{C}\left(-400^{\circ} \mathrm{F}\right)$ y no existiría la vida tal como la conocemos. Esta entrada directa de energía solar también produce otras formas indirectas de energía solar renovable como el viento, la fuerza hidroeléctrica con el agua que cae y fluye y la biomasa con la energía solar convertida en energía química.

Tipos de energías renovables: Las Energías renovables, son un tipo de energía limpia, es decir que excluye cualquier tipo de contaminación, y se generan a partir de recursos naturales renovables como el viento, el agua y el sol. Sin embargo, al día de hoy, aún tienen altos costos tecnológicos, lo que explica porque la mayoría de instalaciones de energías renovables se encuentren en regiones desarrolladas, capaces de invertir en este tipo de tecnología y su respectivo desarrollo.

La energía hidroeléctrica la definen en El aporte de las energías renovables a la situación energética mundial (Farrerons, Olmedo, Caldú y Martínez, 2012).

Energía hidroeléctrica: Está condicionada por el recurso hídrico de cada región. Por lo tanto, aquellas regiones que tengan grandes cantidades de agua y una economía que permita construir las infraestructuras necesarias, serán las que se beneficiarán de este tipo de energía. Aunque se trate de una energía de origen renovable, se debe tener en cuenta que es una tecnología mucho más antigua y consolidada que el resto de las energías renovables que son mucho más actuales.

Energías renovables alternativas: Las energías renovables alternativas son el producto del desarrollo tecnológico que permite aprovechar las fuentes no convencionales y reemplazar las utilizadas desde hace mucho tiempo atrás.

El uso de la energía hidroeléctrica a pequeña escala también constituye una fuente de energía alternativa conocida como Pequeñas Centrales Hidroeléctrica (PCH).

La energía eólica, la energía a partir de la biomasa y la cogeneración están definidas en Análisis costo beneficio de energías renovables no convencionales en Colombia (García, Corredor, Calderón, Gómez, 2013, pg. 46 - 47).

Energía Eólica: Este tipo de centrales usan el viento como fuente de energía, por lo que es necesario ubicarlas en zonas en donde las condiciones geográficas y del viento sean adecuadas para garantizar el máximo rendimiento posible. Los molinos recogen la fuerza del viento (en sus aspas) y un generador se encarga de convertir la energía mecánica en energía eléctrica.

Biomasa/Cogeneración: La biomasa obtiene su energía a partir del sol cuando las plantas están en crecimiento. Las plantas convierten la energía solar en energía química en el proceso de fotosíntesis. Esta energía se libera en forma de calor cuando la biomasa es quemada. El calor producido en el proceso se usa para calentar agua y generar vapor que hace 
girar una turbina para crear electricidad. En algunos casos, la biomasa es quemada en combinación con carbón y se usa para reducir las emisiones de la producción de carbón tradicional.

La energía solar la describe en Energías Renovables y Conservación de Energía (Badii, Guillen \& Abreu, 2016; pag 142).

Energía solar: Mayoría de la energía renovable se deriva directamente o indirectamente desde el sol. Se puede usar la energía solar de forma directa para calentar e iluminar los edificios, para generar electricidad y para calentar el agua, enfriar solar y una variedad de usos comerciales e industriales. El calor del sol también dinamiza los vientos cuya energía se captura por las turbinas del viento. El viento y el calor del sol ocasionan la evaporación del agua. En su artículo Desarrollo de la energía solar en Colombia y sus perspectivas (Humberto Rodríguez Murcia, 2009, pag 2) explica las ventajas y desventajas de la energía solar.

Energía solar: La energía solar — esto es la energía radiante del sol recibida en la tierra - es una fuente de energía que tiene varias importantes ventajas sobre otras y que, para su aprovechamiento, también presenta varias dificultades. Entre sus ventajas se destacan principalmente su naturaleza inagotable, renovable y su utilización libre de polución. Pero, para su utilización, es necesario tener en cuenta su naturaleza intermitente, su variabilidad fuera del control del hombre y su baja densidad de potencia. Estas dificultades conllevan entonces la necesidad de transformarla a otra forma de energía para su almacenamiento y posterior uso

La energía renovable como un negocio rentable: El crecimiento de la demanda de energía es dramático, en la figura 1 se presenta el crecimiento en Latinoamérica con un panorama temporal de 60 años donde e los primeros 25 años el crecimiento fue del $217 \%$ y para los siguientes 35 años se espera que sea del orden del $547 \%$.

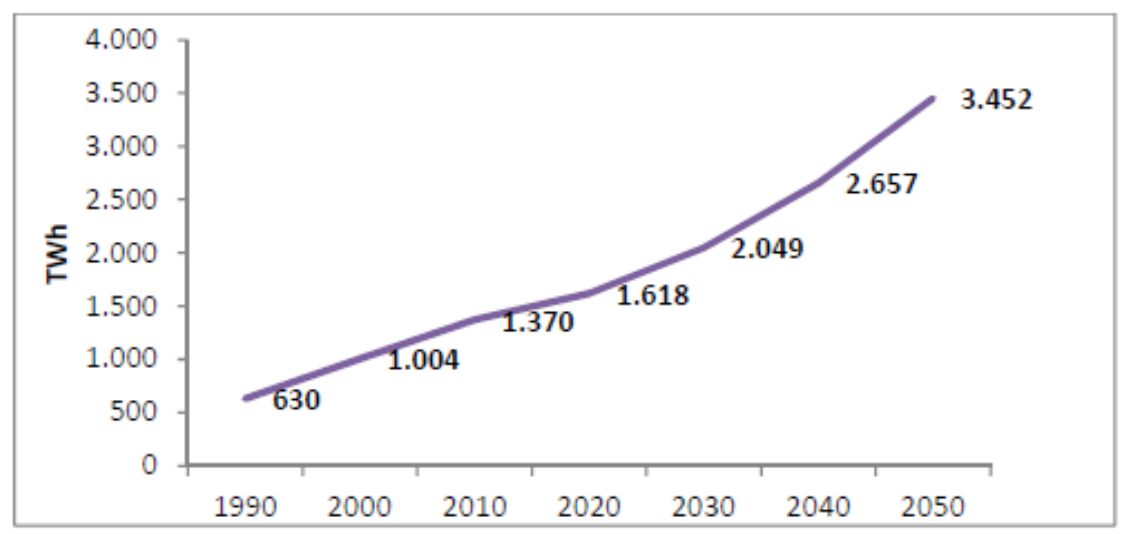

Figura 1. Demanda de energía eléctrica en Latinoamérica 1990 - 2050.

Rethinking Our Energy Future (Vergara, Alatorre, Alves, 2013, pag7) 
Para poder atender esta demanda de manera sostenible se requieren acciones en dos frentes: la primera es racionalizar el uso de la energía mediante la concientización de la sociedad y el uso de tecnologías en los aparatos de consumos masivo para hacerlos más eficientes y de otra parte la utilización sostenible de los recursos energéticos empleando nuevas fuentes como las energías alternativas.

La implantación de estas nuevas tecnologías está teniendo un buen grado de aceptación a nivel mundial, aumentando año tras año las inversiones en el sector de las energías renovables y aumentando la potencia instalada total de cada una de ellas.

En la siguiente tabla se agruparán los indicadores más relevantes de las energías renovables a nivel mundial que demuestran la positiva evolución que tienen estas tecnologías:

Tabla 1. Principales indicadores de las energías renovables.

\begin{tabular}{|c|c|c|c|c|}
\hline Indicador & Unidad & 2009 & 2010 & 2011 \\
\hline Inversión anual en energías renovables & Billones USD & 161 & 220 & 257 \\
\hline $\begin{array}{l}\text { Capacidad total de energías renovables (sin incluir la } \\
\text { hidráulica) }\end{array}$ & GW & 250 & 315 & 390 \\
\hline $\begin{array}{l}\text { Capacidad total de energías renovables (incluyendo la } \\
\text { hidráulica) }\end{array}$ & GW & 1.170 & 1.260 & 1.360 \\
\hline Capacidad total de energías solar fotovoltaica & GW & 23 & 40 & 70 \\
\hline $\begin{array}{l}\text { Capacidad total de energía solar térmica por } \\
\text { concentración }\end{array}$ & GW & 0,7 & 1,3 & 1,8 \\
\hline Capacidad energía eólica & GW & 159 & 198 & 238 \\
\hline Producción de Etanol & $\begin{array}{l}\text { Billones de } \\
\text { litros }\end{array}$ & 73,1 & 86,5 & 86,1 \\
\hline Producción de Biodiesel & $\begin{array}{l}\text { Billones de } \\
\text { litros }\end{array}$ & 17,8 & 18,5 & 21,4 \\
\hline
\end{tabular}

Tomada de El aporte de las energías renovables a la situación energética mundial (Farrerons et al, 2012, pag 51).

Tal y como se ve en la tabla anterior, la inversión en energías renovables a nivel mundial aumenta anualmente, así como la potencia instalada de cada una de ellas y la producción de 
biocombustibles. Pese a este aumento, este tipo de energía aun representa una fracción casi inapreciable en el mix con la energía convencional, por lo que debe ir afianzándose año tras año ganando paulatinamente importancia en la producción de energía.

Adicionalmente esta situación se ratifica en Latinoamérica en el Análisis del mercado de energías renovables en América Latina (Amin, 2016, Pág. 3.), en el que el Director General del IRENA plantea cómo el mercado de energías renovables de la región es uno de los más dinámicos del mundo, apoyado principalmente en la energía hidroeléctrica y en los biocombustibles líquidos, gracias a la determinación de Brasil por diversificar su mix de combustibles para el transporte.

Desde el 2004 la inversión en energías renovables (excluyendo la energía hidroeléctrica), se ha multiplicado por 11 en la región, comparado con seis a nivel mundial. Las tendencias de la inversión avalan la rápida evolución del mix energético de región hacia un conjunto de tecnologías y países más diversificados. En 2015, por primera vez México y Chile se sumaron a Brasil en la lista de los 10 principales mercados de energía renovables del mundo.

Es claro que la necesidad de asegurar el suministro de energía ha influido notablemente en la diversificación energética, promoviendo el desarrollo de las energías alternativas en América Latina, buscando minimizar los efectos económicos negativos por la dependencia de los combustibles fósiles y el impacto de continuos eventos climáticos que influyen en la generación hidráulica, así como la disminución del uso del carbón.

Estas iniciativas se han visto apoyadas por los desarrollos tecnológicos que han producido una disminución drástica de los precios ofertados en las subastas de energía solar y de manera similar está sucediendo con la energía eólica, como se puede apreciar en la figura 2:

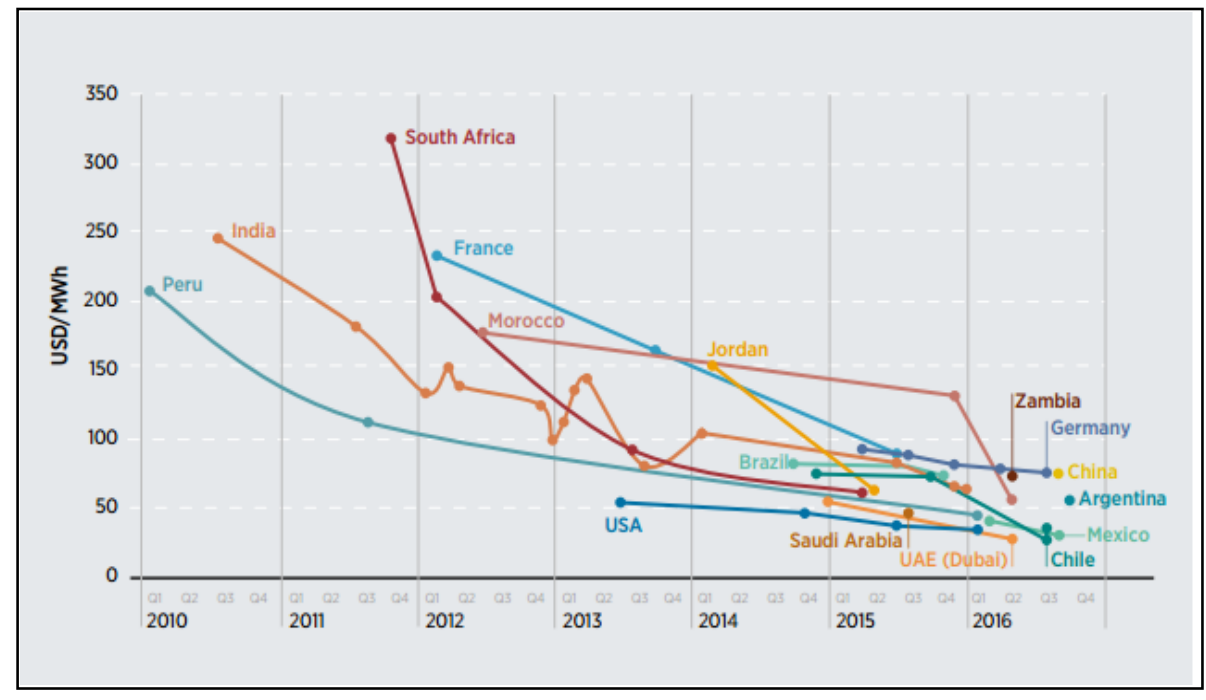

Figura 2. Evolución del precio promedio de energía solar en subastas. Renewable energy auctions analysing 2016 (Irena, 2016, pág 9) 
Fuentes de energía en Colombia: Colombia es una pais con recursos energeticos muy diversos, pero poco desarrollados y concentrados a nivel de generación con un 69\% en energia hidroelectrica, seguida por un $10 \%$ de energia térmica a base de gas y $9 \%$ de energia térmica a base de carbón como se observa en la figura 3 .

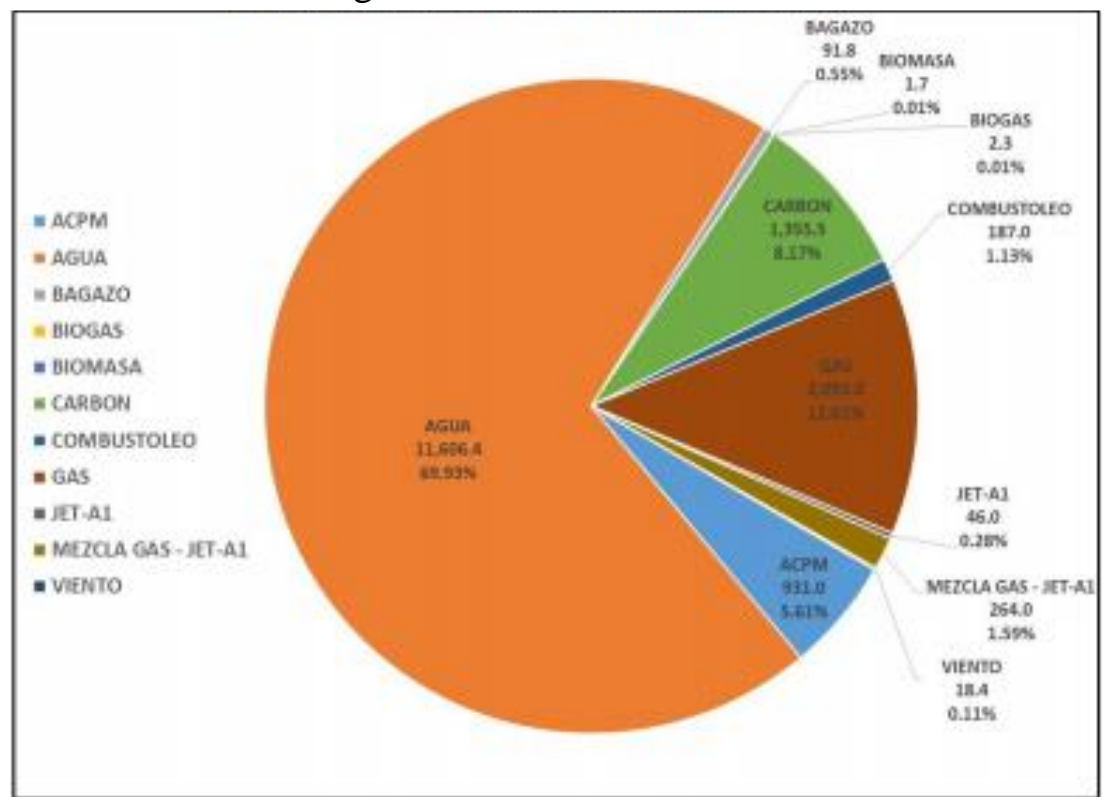

Figura 3. Participación por tecnología en la matriz eléctrica colombiana Informe de variables de generación y del mercado eléctrico (Upme, 2016, pag 1).

Energías Alternativas en Colombia: Colombia tienen grandes ventajas a nivel energético por estar ubicado en la zona ecuatorial y además por contar con climas y ecosistemas variados favoreciendo la generación de energía a partir de fuentes alternativas derivadas del viento, el sol y de los residuos de biomasa como los de la caña de azúcar, aceite de palma, arroz y plátano.

La energía eólica presenta un gran potencial para Colombia en el departamento de La Guajira y la parte costera de la región Caribe, de Santander y Norte de Santander, zonas específicas de Risaralda y Tolima, el Valle del Cauca, el Huila y Boyacá cuentan con recursos aprovechables con mayor potencial de Sur América.

Los vientos de la Guajira durante todo el año con velocidades promedio cercanas a los 9 $\mathrm{m} / \mathrm{s}$ (a $80 \mathrm{~m}$ de altura), y dirección prevalente este-oeste los cuales se estiman representan un potencial energético que se puede traducir en una capacidad instalable del orden de $18 \mathrm{GW}$ eléctricos, es decir, casi 1,2 veces la capacidad de generación instalada en el SIN a diciembre de 2014 (15.465 MW). El potencial de toda la región Caribe colombiana ascendería a una capacidad instalada de $20 \mathrm{GW}$. El poder del viento (Alvaro Pinilla, 2008, pág 68).

Hoy existe el proyecto parque eólico de Jepirachi (19,5 MW de capacidad nominal) debido a la falta de la infraestructura eléctrica necesaria para desalojar la energía producida hacia el 
interior del país, la ausencia de un marco normativo y regulatorio que viabilice la participación de este tipo de energía.

El recurso eólico de La Guajira podría sustituir al gas natural en la generación de energía eléctrica y en la generación de energía por las térmicas que operan con gas y líquidos en la región Caribe. La complementariedad de la eólica con la hídrica se basa en la disposición todo el año de vientos versus la precipitación volátil por el clima como el fenómeno de El Niño y los períodos cíclicos naturales de lluvias y sequía.

En cuanto a energía solar en Colombia. Durante el año de 1992, el antiguo HIMAT y el INEA realizaron el primer Atlas de radiación solar de Colombia, tomando series anuales durante el periodo de 1980 a 1990, de 203 estaciones. Colombia tiene una irradiación promedio de 4,5 $\mathrm{kWh} / \mathrm{m} 2$ /d (UPME, IDEAM, 2005), la cual supera el promedio mundial de $3,9 \mathrm{kWh} / \mathrm{m} 2 / \mathrm{d}$. Las zonas del país como La Guajira y la Costa Atlántica presentan niveles de radiación por encima del promedio nacional que pueden llegar al orden de los $6,0 \mathrm{kWh} / \mathrm{m} 2 / \mathrm{d}$, recurso comparable con algunas de las regiones con mejor recurso en el mundo como es el caso del desierto de Atacama en Chile o los estados de Arizona y Nuevo México en Estados Unidos. El poder del viento (Alvaro Pinilla, 2008, pág 68).

En general, el potencial solar en el país es alto, y tiene la enorme ventaja de que la radiación solar es uniforme durante el año, la zona del Magdalena, la Guajira y San Andrés y Providencia tienen son las de mayor radiación y le siguen la zona de los departamentos de Casanare, Arauca, Guainía, Guaviare, Amazonas, Putumayo y Vaupés.

Por otra parte, la considerable disminución de los costos de los módulos o celdas solares fotovoltaicas han llevado a que sea muy competitivo el mercado minorista de energía eléctrica, especialmente a niveles comercial y residencial.

Mediante la implementación y masificación de pequeños sistemas de autogeneración distribuida se pueden lograr impactos positivos, como son el permitir a los usuarios generar su propia energía, reduciendo así el riesgo de los usuarios a estar sometidos a cierta volatilidad y usuales incrementos en los costos de electricidad.

De otra parte, la Ley 1715 de 2014 introduce la posibilidad de manejar un esquema de créditos para sistemas de autogeneración de pequeña escala, este nuevo escenario seguro propiciará el desarrollo de un mayor número de proyectos dado que la entrega de excedentes representará ahorros o eventualmente ingresos al usuario que harán económicamente más atractiva su inversión es este tipo de sistemas.

En Colombia, la generación total a partir de la biomasa fue de $62.200 \mathrm{GWh}$ de electricidad producidos en 2013, unos $804 \mathrm{GWh}$, el 1,3\%, por el uso del bagazo de la caña de azúcar. Entre tanto, el uso de la biomasa para la producción de calor en la industria, especialmente representado por el uso de bagazo, algo de leña, carbón vegetal y otros residuos (como los de la palma de aceite y el arroz, utilizados de manera general para producción exclusiva de calor), 
representa aproximadamente un $11 \%$ del total de energía final utilizada por tal sector de consumo.

Las demás fuentes de energías alternativas en Colombia como la geotérmica, mareomotriz, entre otras son de muy bajo interés y no se prevé un desarrollo tan importante como el de las demás fuentes alternativas que ya se han analizado.

Conveniencia de la transición en Colombia: Vale destacar los beneficios económicos de conservación de energía que se señalan en el artículo Energías renovables y conservación de energía (Badii et al, 2016, pg. 147 - 148).

Aunque el énfasis se hace para el caso mexicano bien podrían tomarse estos elementos para el estudio de este tipo de energías en Colombia:

1. Reducción de dependencia en los recursos energéticos no renovables: Basado en la cantidad conocida de las reservas energéticas y el consumo actual de estos combustibles se estiman que los combustibles de tipo de petróleo y de gas natural se terminarán en el presente siglo y solamente, el carbón puede durará hasta un par de siglos más.

2. Seguridad nacional y dependencia: La conservación de energía incrementa la seguridad nacional y reduce la dependencia en las fuentes extranjeras.

3. Estabilidad económica: La conservación de energía protege la economía y los consumidores en contra de las posibles fluctuaciones en el precio y la interrupción en el servicio debido a los desastres naturales y otros tipos de desastres.

4. Búsqueda de nuevas fuentes de energía: El creciente demanda para la electricidad y el gas natural requiere la capacidad de encontrar nuevos suministros y fuentes de energía, además, la mayoría de los nuevos suministros requieren mayor cantidad de dinero para invertir lo cual ocasiona aumento en el precio de los combustibles.

5. Economía local: El uso eficiente de energía por la población y el Estado apoya a la economía local y reduce la dependencia en las fuentes extranjeras de petróleo y el gas natural.

6. Economía doméstica: Los programas de uso eficiente de energía proveen mejorías domesticas que a su vez incrementan el confort y bien estar y esto consecuentemente, ocasiona incremento económico en el valor para los dueños de los hogares y también para los negocios.

7. Costos y financiamiento: la inversión es más alta que en el caso de tecnologías convencionales. Así como la falta de un financiamiento especial con intereses muy bajos que estimulen las inversiones.

8. La energía convencional: la posición dominante de algunos agentes del mercado que al contar con portafolios de tecnologías convencionales que han significado cuantiosas inversiones, dificultan la entrada de nuevas tecnologías que les compitan con posibilidad de llegar a ser más eficientes y costo efectivas. 
9. Competencia imperfecta: se requiere desarrollar mecanismos regulatorios que permitan a los agentes pequeños y grandes competir en igualdad de condiciones con estas energías frente a las energías convencionales.

10. Capital humano: la formación, capacidades y experiencia adecuadas. Se requiere contar con personas con conocimientos técnicos y profesionales, en capacidad de difundirlos, multiplicarlos y a la vez implementarlos a través del diseño, estructuración, desarrollo, construcción, operación y mantenimiento de proyectos, equipos y tecnologías. Programas académicos de formación superior, técnica y universitaria que aborden aquellas tecnologías en vías de desarrollo.

Con base en los anteriores puntos en el caso colombiano se evidencia la conveniencia de la transición al uso de energías renovables y especialmente a las energías alternativas. Colombia puede considerar la estructuración, en un horizonte de muy largo plazo, de una economía que se transforme de la actual dependencia en el petróleo (no solo en el aspecto energético sino igualmente en el consumo de derivados de este recurso como materias primas) y la energía hidroeléctrica hacia una economía que pueda disminuir tal dependencia con otros recursos energéticos de origen renovable entre los que la energía eólica, la energía solar y la biomasa sería el sustituto directo del petróleo. Para considerar cómo ir avanzando en esa dirección se pueden mirar los esquemas de los países vecinos que ya presentan avances en la materia como México, Brasil y Chile.

Oportunidades de negocio en Colombia: A partir del análisis de la información expuesta a lo largo del presente artículo se evidencia la oportunidad de negocio para los generadores de energía eléctrica, motivada por la necesidad de la diversificación de la matriz energética hacia fuentes sostenibles más eficientes que soporten el crecimiento de la demanda energética de los próximos años. Los principales nichos de negocio son la energía eólica, la energía solar fotovoltaica y la energía producida a partir de la biomasa.

Indudablemente los cambios en la generación de energía hacia fuentes sustentables obligadas principalmente por un cambio de mentalidad de los consumidores por su mayor consciencia medio ambiental y de conservación del planeta y por la disminución de los costos de las tecnologías, como lo es el caso de paneles solares, crean oportunidades para actores que no se encontraban en el mercado comercial y obligan también a pensar en cambios en otros campos como la construcción con edificaciones amigables con el ambiente (diseño bioclimático) y de bajo consumo energético, el transporte con vehículos eléctricos e híbridos, la iluminación natural y el uso de elementos eficientes como los bombillos led, la educación para la formación de nuevas profesiones y especialidades que hoy no existen. Así lo explica el articulo Ciudades sustentables. Retos y oportunidades (Dania González, 2011, pág 70 y 71)

\section{Conclusión:}


Con base en la revisión de información y análisis de la misma realizada mediante este artículo, es un hecho que la tendencia hacia las energías renovables es irreversible, por la extinción paulatina de los recursos fósiles y por los efectos negativos que su uso como combustible tienen para el planeta y por la mejora permanente en la relación costo beneficio.

Colombia ya está en el camino de la implementación de estas tecnologías y la generación a través de energías alternativas es cada vez más viable, constituyendo una de las mejores soluciones para detener el cambio climático global, además en Colombia contamos con una gran riqueza de recursos que permiten diversificar la matriz de energía para garantizar la estabilidad del suministro energético.

La inversión en proyectos de energía alternativa tiene grandes impactos en la economía del país y particularmente aporta a: la reducción de restricciones en los recursos energéticos, la seguridad nacional, la estabilidad económica del país, el desarrollo tecnológico en la búsqueda e integración de nuevas fuentes de energía, el impacto en la economía local y la economía de los hogares, la inversión extranjera, la eliminación de posiciones dominantes de algunos agentes del mercado y la generación de empleo.

Adicionalmente la implementación de generación con energías alternativas impacta fuertemente la económica nacional impulsando el desarrollo de nuevos nichos de mercado en el sector residencial y comercial a partir de productos y servicios innovadores en los sectores de la construcción, el transporte, la industria, la educación, el empleo y la salud.

De otra parte hay que resaltar que las acciones emprendidas por el gobierno nacional hasta ahora no han sido significativas respecto al potencial de recursos y la misma necesidad de diversificación tecnológica para capturar todos los beneficios derivados de la diversidad energética del país y el potencial de desarrollo a partir de la energía eólica, la energía solar y la biomasa para garantizar el crecimiento sostenible del sector energético como uno de los elementos principales para el desarrollo económico del país.

Este artículo puede dar origen a la realización de otros análisis complementarios como la revisión de la legislación colombiana actual para verificar la adecuada orientación de las políticas públicas y la regulación hacia la promoción de la generación a partir de energías alternativas para beneficio de los sectores industrial y residencial.

También se puede desprender otro análisis respecto a las necesidades de mano de obra del país para las actividades asociadas a las energías alternativas, el nivel de cubrimiento actual y las acciones necesarias a implementar en el sector de la educación y la salud y el trabajo. 


\section{Referencias bibliográficas}

Altamonte, Hugo, Coviello, Manlio, Lutz, Wolfgang, (2003). Energías renovables y eficiencia energética en América Latina y el Caribe. Restricciones y perspectivas.

Arjona Fuentes, Juan Manuel, Hernández Román, Ana, Hidalgo, Luis Amador, (2016). ENERGÍAS RENOVABLES Y DESARROLLO. ANÁLISIS DE LA COOPERACIÓN INTERNACIONAL.

Bueno López, Maximiliano, Rodríguez Sarmiento, Luis Carlos, Rodríguez Sanchez, Patricia Jissette, (2016). Análisis de costos de la generación eléctrica mediante fuentes renovables en el sistema eléctrico colombiano.

Cadena, Angela Ines, (2008). Fuentes energéticas alternativas.

Cadena, Angela Inés, (2012). Requisitos y propuestas para el desarrollo de las fuentes de energías nuevas y renovables en Colombia.

Cadena, Angela, Sergio, Botero, Tautiva, Camilo, Betancur, Luis, Vesga, Daniel, (2008). Regulación para incentivar las energías alternas y la generación distribuida en Colombia.

Camargo, Luis Alejandro, Arboleda, Maria Nohemi, Cardona Adinson, (2014). Producción de energía limpia en Colombia la base para un crecimiento sostenible.

Elizalde Carranza, Miguel, (2016). La agencia internacional para las energías renovables: promesa institucional ante los desafíos energéticos del siglo XXI.

Farrerons Vidal, Oscar, Olmedo Torre, Noelia, Caldú Fogued, Eduardo, Martínez Pérez, Javier, (2012). El aporte de las energías renovables a la situación energética mundial

Garcia, Helena, Corredor, Alejandra, Calderon, Laura, Gómez, Miguel, (2013). Análisis costo beneficio de energías renovables no convencionales en Colombia.

Gonzalez Estrada, Tomás, Valencia Marin, Jorge Alberto, (2015). Integración de las energías renovables no convencionales en Colombia.

Gonzalez, Diana, (2011). Ciudades sustentables. Retos y oportunidades.

Gualteros, Martha Viviana, Hurtado, Enrique, (2013). Revisión de las regulaciones e incentivos para el uso de las energías renovables en Colombia.

Guillen, Badii, Abreu JL, (2016). Energías renovables y conservación de energía. 
Irena, (2016). Renewable energy auctions: Analysis 2016 executive summary. IRENA, Abu Dhabi.

Jiménez Castilla, Tania, (2014). Energías renovables y turismo comunitario: una apuesta conjunta para el desarrollo humano sostenible de las comunidades rurales.

Maya Ochoa, Cecilia, Hernandez Betancur, Juan David, Gallego Munera, Óscar Mauricio, (2012). La valoración de proyectos de energía eólica en Colombia bajo el enfoque de opciones reales.

Monroy, Néstor, Aguirre, Alejando, (2012). El protocolo de Kyoto: ¿Una oportunidad para la industria colombiana?

Morales Ledesma, Patricia, (2007). El sector energético colombiano y las energías renovables.

Morales Sánchez, Carlos Fernando, (2011). Calculo de una tarifa de alimentación para instalaciones renovables.

Ortiz Motta, Diana Carolina, Sabogal Aguilar, Javier, Hurtado Aguirre, Enrique, (2012). Una revisión a la reglamentación e incentivos. De las energías renovables en Colombia.

Pérez Zapata, Jaime, (2017). Identificación y Cuantificación del Potencial de Energía Undimotriz en la costa del departamento del Atlántico, Colombia.

Pinilla, A., (2008). El poder del viento. Revista de ingeniería. Universidad de los Andes. Bogotá. (28)

Prías Caicedo, Omar, Montaña, Diana, (2014). Modelo estratégico de innovación para impulsar la gestión energética en Colombia.

Rodriguez Murcia, Humberto, (2008). Desarrollo de la energía solar en Colombia.

UPME, (2016). Informe mensual de variables de generación y del mercado eléctrico colombiano. Subdirección de energía eléctrica. Grupo de generación.

Vega Clavijo, Lili Tatiana, Prías Caicedo, Omar Fredy, Sierrra Vanegas Fabio Emiro, (2016). Matriz de selección de energías alternativas basada en análisis múltiples atributos para conservación de pescado.

Vergara, Walter, Alatorre, Claudio, Alves, Leandro, (2013). Rethinking our energy future.

Vergara, Walter, Isbell, Paul, Rios, Ana, Gomez, José Ramón, Alves, Leandro, (2014). Beneficios para la sociedad de la adopción de Fuentes renovables de energía en América Latina y el Caribe. 